\title{
Topologically distinct classes of valence-bond solid states with their parent Hamiltonians
}

\author{
Hong-Hao Tu and Guang-Ming Zhang* \\ Department of Physics, Tsinghua University, Beijing 100084, China
}

Tao Xiang

Institute of Physics, Chinese Academy of Sciences, P.O. Box 603, Beijing 100190, China

and Institute of Theoretical Physics, Chinese Academy of Sciences, P.O. Box 2735, Beijing 100190, China

Zheng-Xin Liu and Tai-Kai Ng

Department of Physics, Hong Kong University of Science and Technology, Kowloon, Hong Kong, China

(Received 6 April 2009; revised manuscript received 19 May 2009; published 1 July 2009)

\begin{abstract}
We present a general method to construct one-dimensional translationally invariant valence-bond solid states with a built-in Lie group $G$ and derive their matrix product representations. The general strategies to find their parent Hamiltonians are provided so that the valence-bond solid states are their unique ground states. For quantum integer-spin- $S$ chains, we discuss two topologically distinct classes of valence-bond solid states: one consists of two virtual $S U(2)$ spin- $J$ variables in each site and another is formed by using two $S O(2 S+1)$ spinors. Among them, a spin-1 fermionic valence-bond solid state, its parent Hamiltonian, and its properties are discussed in detail. Moreover, two types of valence-bond solid states with $S O(5)$ symmetries are further generalized and their respective properties are analyzed as well.
\end{abstract}

DOI: 10.1103/PhysRevB.80.014401

PACS number(s): 75.10.Pq, 75.10.Jm, 03.65.Fd

\section{INTRODUCTION}

In recent years, the study of topological order has become a common issue in condensed-matter physics and quantum information theory. ${ }^{1-7}$ Historically, this concept was proposed to describe fractional quantum Hall states, ${ }^{8}$ which are incompressible quantum liquids with a finite-energy gap to all bulk excitations. These new quantum phases of matter cannot be described by a local-order parameter with spontaneous symmetry breaking. So the discovery of fractional quantum Hall effect brings great challenge to the GinzburgLandau theory, which is a cornerstone paradigm to characterize phases and phase transitions in condensed-matter physics. From the viewpoint of quantum information theory, the appearance of a long-range quantum entanglement plays an essential role in the topologically ordered states. However, a general multipartite entanglement measure that captures the most relevant physical properties is still lacking because the number of parameters required to describe a quantum many-body state usually grows exponentially with the particle number.

The topological order appears not only in the twodimensional fractional quantum Hall states but also in onedimensional (1D) systems, for instance, the quantum integerspin chains. In 1983, Haldane ${ }^{9}$ predicted that quantum integer-spin antiferromagnetic Heisenberg chains have an exotic energy gap. Later, Affleck, Kennedy, Lieb, and Tasaki (AKLT) (Ref. 10) found a family of exactly solvable integerspin models with valence-bond solid (VBS) ground states, and the presence of an excitation gap can be proved rigorously. In a spin-coherent-state representation, these VBS states share a striking analogy to the fractional quantum Hall states. ${ }^{11}$ Although the two-point spin correlation decays exponentially, den Nijs and Rommelse ${ }^{12}$ found a hidden topological order in the $S=1$ VBS state, which is characterized by nonlocal string order parameters. For the $S=1$ VBS state, the long-range string order and the fourfold degeneracy in an open chain can be understood as natural consequences of a hidden $Z_{2} \times Z_{2}$ symmetry breaking. ${ }^{13,14}$ For the standard integer-spin Heisenberg models, the existence of spin- $S / 2$ edge states was also verified by quantum field-theory mappings ${ }^{15}$ and numerical calculations, ${ }^{16}$ which coincide with the VBS picture of the AKLT models. Experimentally, the VBS picture for $S=1$ Haldane chain was supported by the electron-spin-resonance studies ${ }^{17,18}$ of the compound $\mathrm{Ni}\left(\mathrm{C}_{2} \mathrm{H}_{8} \mathrm{~N}_{2}\right)_{2} \mathrm{NO}_{2}\left(\mathrm{ClO}_{4}\right)$ (NENP), the NMR imaging, ${ }^{19}$ and the magnetic neutron-scattering study ${ }^{20}$ of the quasi-onedimensional material $\mathrm{Y}_{2} \mathrm{BaNiO}_{5}$.

The one-dimensional VBS states can be represented in a matrix product form. ${ }^{21-23}$ Moreover, it was found ${ }^{24}$ that the density-matrix renormalization group, ${ }^{25}$ the most powerful numerical method for one-dimensional quantum systems, converges to a matrix product wave function as its fixed point. This important observation stimulates the formulation of the numerical techniques in one dimension by using the matrix product variational wave functions. ${ }^{26}$ From a quantum information perspective, the validity of the matrix product variational ansatz depends on whether the true quantum ground states of the system obey an area law of entanglement entropy. ${ }^{27}$ In this sense, the VBS states are only slightly entangled because their entanglement entropies have upper bounds even in the thermodynamic limit. Recently, these VBS states have received considerable attention since they provide a playground to test the proposed measures of multipartite entanglement. ${ }^{28-30}$ Toward the potential applications, it was suggested that the VBS states ensure measurement-based quantum computation. ${ }^{31}$ For the $S=1$ VBS state of the AKLT model, Brennen and Miyake ${ }^{32}$ have shown that a gap-protected measurement-based quantum computation can be performed within the degenerate ground states. 
In this paper, we will introduce one-dimensional translationally invariant VBS states with a built-in Lie group $G$ and present a general method to construct their parent Hamiltonians. For quantum integer-spin- $S$ chains, we focus on two classes of VBS states. The local spin- $S$ states are formed by two virtual $S U(2)$ spin- $J$ variables in the first class and by two $S O(2 S+1)$ spinors in the second one. To illustrate the method, we choose the $S=1$ fermionic VBS state with virtual spin $J=1$ as an explicit example to find the parent Hamiltonian. Apart from the $S=1$ VBS state with virtual spin $J$ $=1 / 2$ and the $S=2$ VBS state with virtual spin $J=3 / 2$ as the intersection elements, the VBS states of the two classes are shown to be topologically distinct to each other, which can be characterized by their edge spin representations in open chain systems. We also apply our method to investigate $S O(5)$ symmetric spin chains and discuss several VBS states with interesting properties.

The outline of this paper is structured as follows. In Sec. II, we will introduce VBS states with a Lie group symmetry and derive their matrix product representations. In Sec. III, we will focus on quantum integer-spin chains and study two topologically distinct classes of VBS states including spin- $S$ VBS states formed by virtual $S U(2)$ spin- $J$ particles and by virtual $S O(2 S+1)$ spinor particles. Moreover, a spin-1 fermionic VBS state is extensively studied as an example and we construct its parent Hamiltonian explicitly. Section IV is devoted to the $S O(5)$ symmetric VBS states and their physical properties. In Sec. V, some conclusions are drawn.

\section{GENERAL CONSTRUCTION OF VBS STATES}

\section{A. Matrix product form}

We begin with a quantum one-dimensional chain with $N$ lattice sites. In each site, the states $\{|m\rangle\}(m=1, \ldots, d)$ transform under a $d$-dimensional irreducible representation (IR) $G_{d}$ of a Lie group $G$. Let us imagine that the physical Hilbert space is formed by two virtual identical particles, whose internal quantum numbers $\{|\alpha\rangle\}(\alpha=1, \ldots, D)$ transform under the $D$-dimensional IR $G_{D}$ of the same Lie group $G$. Here we require that both singlet representation $G_{I}$ and IR $G_{d}$ are included in the tensor product decomposition of two $G_{D}$ 's. The first requirement means that $G_{D}$ is a self-conjugate IR, i.e., the complex-conjugate representation of $G_{D}$ is equivalent to $G_{D}$. The latter requirement can be implemented by the projection operator onto the physical Hilbert space ${ }^{33}$

$$
P=\sum_{m=1}^{d} \sum_{\alpha, \beta=1}^{D} P_{\alpha, \beta}^{[m]}|m\rangle\langle\alpha, \beta|,
$$

where $P_{\alpha, \beta}^{[m]}$ is the Clebsch-Gordan coefficient defined by $P_{\alpha, \beta}^{[m]}=\left\langle G_{d}, m \mid G_{D}, \alpha ; G_{D}, \beta\right\rangle$. For VBS states in a periodic chain, each lattice site forms a valence-bond singlet $|I\rangle$ with its neighboring sites by pairing two virtual particles (see Fig. 1 ). Thus, the wave functions of the VBS states can be expressed as

$$
|\Psi\rangle=\left(\otimes_{k=1}^{N} P_{k, \bar{k}}\right)|I\rangle_{12}^{-}|I\rangle_{23}^{-} \cdots|I\rangle_{\bar{N} 1},
$$

where the valence-bond singlet $|I\rangle$ is given by

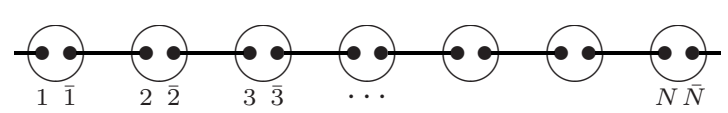

FIG. 1. The schematic of the VBS states with a built-in Lie group $G$. Each dot denotes a virtual particle transforming under $G_{D}$ irreducible representations of Lie group $G$. The solid lines represent valence-bond singlets formed by two virtual $G_{D}$ irreducible representations on the neighboring sites and the circles denote the projections of the virtual particles in each lattice site onto the physical $G_{d}$ irreducible representations.

$$
|I\rangle_{i j}=\sum_{\alpha, \beta=1}^{D} Q_{\alpha, \beta}|\alpha\rangle_{i} \otimes|\beta\rangle_{j}
$$

Here $Q_{\alpha, \beta}=\left\langle G_{I}, I \mid G_{D}, \alpha ; G_{D}, \beta\right\rangle$ is the Clebsch-Gordan coefficient of contracting two virtual $G_{D}$ representations to form a singlet representation $G_{I}$.

In the present formalism, the VBS states can be easily written in a matrix product form. Since $P_{\alpha, \beta}^{[m]}$ and $Q_{\alpha, \beta}$ can be viewed as the matrix elements of $D \times D$ matrices of $P^{[m]}$ and $Q$, the VBS states in Eq. (2) can be thus written as the following matrix product form:

$$
|\Psi\rangle=\sum_{m_{1} \cdots m_{N}} \operatorname{Tr}\left(A^{\left[m_{1}\right]} A^{\left[m_{2}\right]} \cdots A^{\left[m_{N}\right]}\right)\left|m_{1} \cdots m_{N}\right\rangle,
$$

where $A^{[m]}=P^{[m]} Q$ is a $D \times D$ matrix.

In periodic boundary conditions, the VBS states are invariant under lattice translation and transformation of the Lie group $G$ by construction. Although no local order parameters can be found to characterize these states, the $A^{[\mathrm{m}]}$ matrices can fully determine their physical properties and render a "local" description. In open boundary conditions, edge states emerge at the two ends of the chain and then the matrix product form of the VBS states is given by

$$
\left|\Psi_{\alpha, \beta}\right\rangle=\sum_{m_{1} \cdots m_{N}}\left(A^{\left[m_{1}\right]} A^{\left[m_{2}\right]} \cdots A^{\left[m_{N}\right]}\right)_{\alpha, \beta}\left|m_{1} \cdots m_{N}\right\rangle,
$$

where the matrix indices $\alpha$ and $\beta$ denote the edge states. These edge degrees of freedom are described by two fractionalized particles transforming under $G_{D}$ representation of the Lie group $G$. Actually, the edge states and their representations are characteristic features of the VBS states because they fully determine the local $A^{[\mathrm{m}]}$ matrices.

Another useful way to represent the VBS states is to use boson or fermion realization methods. To illustrate this method, we trace back to the tensor product decomposition of IRs of Lie algebras. According to the group theory, the physical states $|m\rangle$ under the exchange of the two identical virtual particles are either symmetric or antisymmetric depending on $G_{d}$ and $G_{D}$. Thus, the two virtual particles with fermion statistics create the antisymmetric states, while the bosonic particles yield the symmetric ones. In some cases, there are still several channels with the same exchange symmetry and additional projection has to be used to single out the physical states in $G_{d}$. For example, the fermionic realization of a spin-2 VBS state with virtual spin $J=3 / 2$ was considered in Ref. 34. In this case, both site-quintet states $(S$ $=2)$ and site-singlet state $(S=0)$ are allowed for two spin-3/2 
fermions on a single site and an extra projection can remove the unphysical site-singlet state. There also exists the Schwinger boson realization that symmetrizes several fundamental IRs to form higher dimensional $G_{d}$ 's. ${ }^{11,35-37}$ In fact, all these boson/fermion realization methods play the role of (sometimes partially) deleting the unphysical states.

\section{B. Parent Hamiltonian: Locating the null space}

Following the spirit of the AKLT model, one can construct the parent Hamiltonians such that the VBS states in Eq. (2) are their unique ground states. It is most convenient to work with the matrix product form. For the matrix product states in Eq. (4), one can readily find that their reduced density matrix $\rho_{l}$ of $l$ successive sites has a rank of $D^{2}$ at most. This suggests that the reduced density matrix $\rho_{l}$ of these VBS states always has null space for sufficient large $l$. These states are annihilated by the local projection operators supported in the null space. Hence, they are always exact zeroenergy ground states of the translationally invariant Hamiltonians

$$
H=\sum_{i} h_{i}
$$

where $h_{i}$ contains a sum of the positive semidefinite projection operators supported in the null space from site $i$ to $i+l$ -1 . Previously, the parent Hamiltonians of the matrix product states for spin-ladder systems had been studied by similar methods. ${ }^{38}$

Let us begin with the simplest cases with only nearestneighbor (NN) interactions. Now the null space can be obtained from the VBS picture of these states. The Hilbert space of two neighboring sites can be divided into a direct sum of different IR channels according to the tensor product decomposition $G_{d} \otimes G_{d}$. Once a valence-bond singlet of two virtual $G_{D}$ 's is formed, the remaining two particles of adjacent sites can transform under a direct sum of IRs resulting from $G_{D} \otimes G_{D}$. Therefore, the IR channels contained in $G_{d}$ $\otimes G_{d}$ but absent in $G_{D} \otimes G_{D}$ constitute the null space in the two-site-reduced density matrix.

The above steps to locate the null space can be embedded in a matrix product formalism. Practically, we rewrite the matrix product states in Eq. (4) as

$$
|\Psi\rangle=\operatorname{Tr}\left(g_{1} g_{2}, \ldots, g_{N}\right),
$$

where the local matrix $g_{i}$ is defined by

$$
g_{i} \equiv \sum_{m_{i}} A^{\left[m_{i}\right]}\left|m_{i}\right\rangle
$$

To locate the null space, we resort to a "coarse-graining" procedure that converts the spins of adjacent sites to block spins. Since the VBS states are invariant under lattice translation, we can block the spins in sites 1 and 2 as

$$
g_{1} g_{2}=\sum_{m_{1}, m_{2}} A^{\left[m_{1}\right]} A^{\left[m_{2}\right]}\left|m_{1}, m_{2}\right\rangle=\sum_{G_{12}, M_{12}^{G}} B^{\left[G_{12}, M_{12}^{G}\right]}\left|G_{12}, M_{12}^{G}\right\rangle,
$$

where the $D \times D$ matrices $B^{\left[G_{12}, M_{12}^{G}\right]}$ are given by

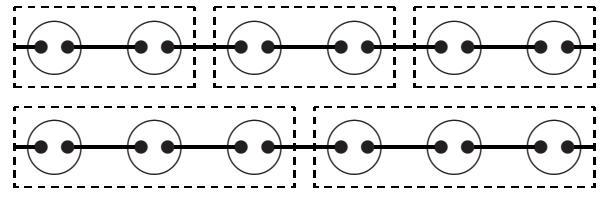

FIG. 2. The schematic of the coarse-graining process that converts the spins of successive sites to block spins. This procedure leads to a matrix product state with block spins and the null space of a block-spin-reduced density matrix can be identified.

$$
B^{\left[G_{12}, M_{12}^{G}\right]}=\sum_{m_{1}, m_{2}} A^{\left[m_{1}\right]} A^{\left[m_{2}\right]}\left\langle G_{12}, M_{12}^{G} \mid G_{d}, m_{1} ; G_{d}, m_{2}\right\rangle .
$$

Here $G_{12}$ 's distinguish the IRs of nearest-neighbor bond spin channels and $\left|G_{12}, M_{12}^{G}\right\rangle$ are the states in IR channel $G_{12}$. Correspondingly, $\left\langle G_{12}, M_{12}^{G} \mid G_{d}, m_{1} ; G_{d}, m_{2}\right\rangle$ is the ClebschGordan coefficient to combine the states of $G_{d}$ 's into the states of $G_{12}$. In the example of $S U(2), G_{12}$ denotes the total bond spin $S_{12}$ and $-S_{12} \leq M_{12}^{S} \leq S_{12}$. After this coarsegraining procedure, the VBS states are transformed to a matrix product form with two-site block spins characterized by the block-independent matrices $B^{\left[G, M^{G}\right]}$. Since the two-site block-spin states $\left|G_{12}, M_{12}^{G}\right\rangle$ form a complete orthogonal set, the null space in the reduced density matrix of a two-site block is given by those IR channels with $B^{\left[G_{12}, M_{12}^{G}\right]}=0$.

The null space for more than two adjacent sites is no longer easily visualized. However, the blocking process of $g$ matrices can be proceeded without any fundamental difficulties (see Fig. 2). In Sec. III B, we will study the spin-1 fermionic VBS state by using this powerful method.

The uniqueness of the VBS ground states of the constructed Hamiltonians has to be further clarified. Generally, the ground-state degeneracy will occur if there exists another state with a larger null space in the reduced density matrix of the present interaction range. To lift the degeneracy, one can locate the null space in an extended range by blocking more spins and modifying the Hamiltonian correspondingly. To justify the uniqueness, it is helpful to numerically diagonalize an open chain Hamiltonian with several lattice sites. If the numerically calculated ground-state degeneracy is $D^{2}$, i.e., the ground states are all contained in the matrix product form, one can prove the uniqueness of VBS ground states by a mathematical induction method. The basic idea is to assume that the VBS states $\left|\Psi_{\alpha, \beta}\right\rangle$ in Eq. (5) are the only ground states of a projector Hamiltonian $H(N)$ of an open chain with $N$ sites. Then, the ground states of an open chain with $N+1$ lattice sites can be written as the following superposition of $\left|\Psi_{\alpha, \beta}\right\rangle$ and $\left|m_{N+1}\right\rangle$ :

$$
\left|\Psi_{N+1}\right\rangle=\sum_{\alpha \beta, m_{N+1}} W_{\beta \alpha, m_{N+1}}\left|\Psi_{\alpha, \beta}\right\rangle \otimes\left|m_{N+1}\right\rangle .
$$

The vectors $\left|m_{N+1}\right\rangle$ on the site $N+1$ decouple from the excited states of $H(N)$ because such a coupling does not gain energy from the projector Hamiltonian $H(N+1)$. Now we can change the notation $W_{\beta \alpha, m_{N+1}} \equiv W_{\beta, \alpha}^{\left[m_{N+1}\right]}$ and then $\left|\Psi_{N+1}\right\rangle$ can be immediately written in a matrix product form 


$$
\left|\Psi_{N+1}\right\rangle=\sum_{m_{1} \cdots m_{N+1}} \operatorname{Tr}\left(A^{\left[m_{1}\right]} \cdots A^{\left[m_{N}\right]} W^{\left[m_{N+1}\right]}\right)\left|m_{1} \cdots m_{N+1}\right\rangle,
$$

where $D \times D$ matrix $W^{\left[m_{N+1}\right]}$ can be fully determined because $\left|\Psi_{N+1}\right\rangle$ are the zero-energy ground states of $H(N+1)$. After solving this eigenvalue problem, one can find that $\left|\Psi_{N+1}\right\rangle$ can be written in the form of Eq. (4). This final step completes the mathematical induction proof. For periodic boundary conditions, the VBS ground state should be a linear combination of the $D^{2}$ states in Eq. (5) and be annihilated by the extra projectors acting on the two ends of the chain. This will lead to the VBS ground state in the form of Eq. (4).

Actually, those matrix product states with $D^{2}$ linearly independent $\left|\Psi_{\alpha, \beta}\right\rangle$ in a finite open chain satisfy the so-called injective property. ${ }^{21,33,39}$ The injectivity of the matrix product states not only ensures the ground-state uniqueness of the parent Hamiltonian but also guarantees the existence of an energy gap and the exponentially decaying correlation functions of local operators.

\section{GENERAL VBS STATES FOR QUANTUM INTEGER- SPIN CHAINS}

In Sec. II, we set up a framework to study the VBS states with a built-in Lie group $G$. To test these abstract formalisms, we begin with the quantum integer-spin- $S$ chains and consider two classes of VBS states. In these two VBS classes, the virtual particles transform under spin- $J$ representations and $2^{S}$-dimensional $S O(2 S+1)$ spinor representations, respectively. Toward the first class, Sanz et al. ${ }^{39}$ have explored $S U(2)$-invariant two-body Hamiltonians which have such states as their eigenstates. In the present work, we further require that these VBS states are unique ground states of the parent Hamiltonians. However, the price we usually have to pay is to include multispin interactions in the parent Hamiltonians. This situation will be treated for the spin-1 fermionic VBS state in Sec. III B. For the second class, we will show that these states are equivalent to the $S O(2 S+1)$ symmetric matrix product states introduced in our previous work. ${ }^{34}$ However, the present formalism explains the origin of the emergent $S O(2 S+1)$ symmetry in these VBS states and shows that their edge states are $S O(2 S+1)$ spinors. Therefore, the two VBS classes are sharply distinct from each other for $S \geq 3$.

\section{A. Spin- $S$ VBS states with virtual spin- $J$ particles}

As a warm up, let us apply the formalism in Sec. II to the spin- $S$ VBS states with two virtual spin- $J$ particles in each site. It is well known that the product of two spin- $J$ representation

$$
J \otimes J=0 \oplus 1 \oplus \cdots \oplus 2 J
$$

always contains a singlet and the physical spin- $S$ representation if $J \geq S / 2$. After replacing the $S U(2)$ Clebsch-Gordan coefficient $P_{\alpha, \beta}^{[m]}=\langle S, m \mid J, \alpha ; J, \beta\rangle$ in Eq. (1) and the spin- $J$ valence-bond singlet

$$
|I\rangle_{i j}=\sum_{\alpha=-J}^{J}(-1)^{J-\alpha}|\alpha\rangle_{i} \otimes|-\alpha\rangle_{j}
$$

in Eq. (3), the $(2 J+1) \times(2 J+1)$ matrix $A^{[m]}$ in Eq. (4) can be written as

$$
A^{[m]}=\sum_{\alpha, \beta}(-1)^{J+\beta}\langle S, m \mid J, \alpha ; J,-\beta\rangle|J, \alpha\rangle\langle J, \beta|,
$$

where $-J \leq \alpha, \beta \leq J$. These $A^{[m]}$ matrices are rank $S$ irreducible spherical tensors and satisfy the following commutation relations:

$$
\begin{gathered}
{\left[J_{z}, A^{[m]}\right]=m A^{[m]},} \\
{\left[J_{ \pm}, A^{[m]}\right]=\sqrt{(S \mp m)(S \pm m+1)} A^{[m \pm 1]},}
\end{gathered}
$$

where $J_{ \pm}$and $J_{z}$ generate the spin- $J$ representation of the $S U(2)$ algebra,

$$
\begin{gathered}
J_{ \pm}=\sum_{\alpha} \sqrt{(J \mp \alpha)(J \pm \alpha+1)}|J, \alpha \pm 1\rangle\langle J, \alpha|, \\
J_{z}=\sum_{\alpha} \alpha|J, \alpha\rangle\langle J, \alpha| .
\end{gathered}
$$

For the celebrated VBS states of the AKLT models, i.e., the case of virtual spin $J=S / 2$, we can also use the Schwinger boson representation to express the VBS states. In the Schwinger boson language, the spin operators are expressed by

$$
S_{i}^{+}=a_{i}^{\dagger} b_{i}, S_{i}^{-}=b_{i}^{\dagger} a_{i}, S_{i}^{z}=\left(a_{i}^{\dagger} a_{i}-b_{i}^{\dagger} b_{i}\right) / 2,
$$

with a local constraint $a_{i}^{\dagger} a_{i}+b_{i}^{\dagger} b_{i}=2 S$. Then, the integer-spin- $S$ VBS states of the AKLT models in a periodic chain are expressed as ${ }^{11}$

$$
|\mathrm{AKLT}\rangle=\prod_{i}\left(a_{i}^{\dagger} b_{i+1}^{\dagger}-b_{i}^{\dagger} a_{i+1}^{\dagger}\right)^{S}|v\rangle,
$$

where $|v\rangle$ is the vacuum with no particle occupation. The matrix product forms of these VBS states are obtained by Totsuka and Suzuki. ${ }^{23}$ Since the null space of two neighboring sites is the total bond spin $S+1, \ldots, 2 S$ channels, the VBS states in Eq. (19) are exact ground states of AKLT Hamiltonians ${ }^{10,11}$

$$
H_{\mathrm{AKLT}}=\sum_{i} \sum_{S_{T}=S+1}^{2 S} J_{S_{T}} P_{S_{T}}(i, i+1),
$$

where all $J_{S_{T}}>0$ and $P_{S_{T}}(i, j)$ is the projection operator on total bond spin channel $S_{T}$. These $S U(2)$ invariant projection operators can be written as polynomials of spin-exchange interactions $\mathbf{S}_{i} \cdot \mathbf{S}_{j}$ up to $2 S$ powers

$$
P_{S_{T}}(i, j)=\prod_{\substack{S^{\prime}=0, S^{\prime} \neq S_{T}}}^{2 S} \frac{2 \mathbf{S}_{i} \cdot \mathbf{S}_{j}+2 S(S+1)-S^{\prime}\left(S^{\prime}+1\right)}{S_{T}\left(S_{T}+1\right)-S^{\prime}\left(S^{\prime}+1\right)} .
$$

For $S / 2<J<S$ cases, at first glance, the null space of two neighboring sites is given by the total bond spin channels $2 J+1, \ldots, 2 S$, which is smaller than the VBS states of AKLT 
model. According to Sec. II B, one may conclude that nextnearest-neighbor interactions are needed to be construct their parent Hamiltonians. However, there is an exception: the VBS states with $S=2$ and $J=3 / 2$. We will discuss this special case in Sec. III C.

\section{B. Spin-1 fermionic VBS states}

Now we consider $S=1$ VBS state with virtual spin $J=1$, which belongs to the class in Sec. III A. Since $S=1$ is the only antisymmetric product of two virtual $J=1$ particles, one can use the fermionic statistics to implement the projection onto the physical $S=1$ subspace. Thus, the physical $S=1$ states are written as

$$
|1\rangle=c_{1}^{\dagger} c_{0}^{\dagger}|v\rangle, \quad|0\rangle=c_{1}^{\dagger} c_{-1}^{\dagger}|v\rangle, \quad|-1\rangle=c_{0}^{\dagger} c_{-1}^{\dagger}|v\rangle .
$$

The $S U(2)$ spin operators are $S_{i}^{a}=\Sigma_{\mu, \nu=1}^{3} c_{i \mu}^{\dagger} S_{\mu \nu}^{a} c_{i \nu}(a=x, y, z)$, where $S^{a}$ are the usual $3 \times 3$ spin-1 matrices. The total spin $\mathbf{S}_{i}^{2}=2$ on each site is imposed by a local constraint $\sum_{\mu=1}^{3} c_{i \mu}^{\dagger} c_{i \mu}=2$. In terms of these fermionic variables, the $S$ $=1$ VBS state with virtual spin $J=1$ can be exactly written as

$$
\left|\Psi_{1}\right\rangle=\prod_{i=1}^{N}\left(c_{i, 1}^{\dagger} c_{i+1,-1}^{\dagger}-c_{i, 0}^{\dagger} c_{i+1,0}^{\dagger}+c_{i,-1}^{\dagger} c_{i+1,1}^{\dagger}\right)|v\rangle,
$$

which has a matrix product form in Eq. (4) with

$$
\begin{gathered}
A^{[1]}=\left(\begin{array}{ccc}
0 & -1 & 0 \\
0 & 0 & -1 \\
0 & 0 & 0
\end{array}\right), \quad A^{[0]}=\left(\begin{array}{ccc}
1 & 0 & 0 \\
0 & 0 & 0 \\
0 & 0 & -1
\end{array}\right), \\
A^{[-1]}=\left(\begin{array}{lll}
0 & 0 & 0 \\
1 & 0 & 0 \\
0 & 1 & 0
\end{array}\right) .
\end{gathered}
$$

Following the method in Sec. II B, we can construct the parent Hamiltonian for this fermionic VBS state. To locate the null space, it is sufficient to block three successive spins. The tensor decomposition of three spin-1 representation yields

$$
\begin{aligned}
1 \otimes 1 \otimes 1 & =(0 \oplus 1 \oplus 2) \otimes 1 \\
& =1 \oplus 0 \oplus 1^{\prime} \oplus 2 \oplus 1^{\prime \prime} \oplus 2^{\prime} \oplus 3,
\end{aligned}
$$

which provides a natural basis for block spins. In this basis, the block states can be denoted by $\left|S_{12} ; S, M\right\rangle$, where $S$ and $M$ are total spin and magnetic quantum number of the three sites and $S_{12}$ is the total spin of the first two sites. For the representations $1^{\prime}$ and 2, we have $S_{12}=1$. For the representations $1^{\prime \prime}$ and $2^{\prime}, S_{12}=2$. For the representations 0 and 3 , the index $S_{12}$ can be suppressed and does not lead to misunderstanding.

After blocking the three spins, we obtain

$$
\begin{aligned}
g_{1} g_{2} g_{3} & =\sum_{m_{1} m_{2} m_{3}} A^{\left[m_{1}\right]} A^{\left[m_{2}\right]} A^{\left[m_{3}\right]}\left|m_{1}, m_{2}, m_{3}\right\rangle \\
& =\sum_{S, M} \sum_{S_{12}} C_{S_{12}^{[S, M]}}^{[S}\left|S_{12} ; S, M\right\rangle,
\end{aligned}
$$

where the $3 \times 3$ matrices $C_{S_{12}}^{[S, M]}$ are given by

$$
\begin{aligned}
C_{S_{12}}^{[S, M]}= & \sum_{m_{1} m_{2} m_{3}}\left\langle S_{12}, m_{1}+m_{2} \mid 1, m_{1} ; 1, m_{2}\right\rangle \\
& \times\left\langle S, M \mid S_{12}, m_{1}+m_{2} ; 1, m_{3}\right\rangle A^{\left[m_{1}\right]} A^{\left[m_{2}\right]} A^{\left[m_{3}\right]} .
\end{aligned}
$$

The matrices $C_{S_{12}}^{[S, M]}$ can be calculated by using Eq. (24). First, we find that $C^{[0,0]} \neq 0$ and $C^{[3, M]}=0$. This means that the spin- 0 singlet state is contained but the spin-3 states are absent in every three-site block. The other nonvanishing matrices $C_{S_{12}}^{[S, M]}$ satisfy the following equations

$$
\begin{gathered}
C_{2}^{[2, M]}=\sqrt{3} C_{1}^{[2, M]}, \\
C_{2}^{[1, M]}=-\sqrt{\frac{5}{3}} C_{1}^{[1, M]}=\frac{\sqrt{5}}{4} C_{0}^{[1, M]} .
\end{gathered}
$$

According to Eq. (26), the unnormalized states contained in the three-site block $g_{1} g_{2} g_{3}$ are one spin- 0 state and three spin-1 states

$$
4|0 ; 1, M\rangle-\sqrt{3}|1 ; 1, M\rangle+\sqrt{5}|2 ; 1, M\rangle
$$

with $-1 \leq M \leq 1$ and five spin-2 states

$$
|1 ; 2, M\rangle+\sqrt{3}|2 ; 2, M\rangle
$$

with $-2 \leq M \leq 2$. By using the Gram-Schmidt orthogonalization method, we find that seven spin-3 states $|3, M\rangle$ with $-3 \leq M \leq 3$, five spin-2 states

$$
\left|\phi_{2, M}\right\rangle=\sqrt{3}|1 ; 2, M\rangle-|2 ; 2, M\rangle
$$

with $-2 \leq M \leq 2$, six spin-1 states

$$
\begin{gathered}
\left|\phi_{1, M}\right\rangle=\sqrt{3}|0 ; 1, M\rangle+4|1 ; 1, M\rangle, \\
\left|\varphi_{1, M}\right\rangle=4|0 ; 1, M\rangle-\sqrt{3}|1 ; 1, M\rangle-\frac{19}{\sqrt{5}}|2 ; 1, M\rangle
\end{gathered}
$$

with $-1 \leq M \leq 1$ span the null space in the reduced density matrix of the three-site block. Therefore, the three-site projector Hamiltonian for which the spin-1 fermionic VBS state is the zero-energy ground state is thus given by

$$
\begin{aligned}
h= & \lambda_{3} \sum_{|M| \leq 3}|3, M\rangle\left\langle 3, M\left|+\lambda_{2} \sum_{|M| \leq 2}\right| \phi_{2, M}\right\rangle\left\langle\phi_{2, M}\right| \\
& +\sum_{|M| \leq 1}\left(\lambda_{1}\left|\phi_{1, M}\right\rangle\left\langle\phi_{1, M}\left|+\lambda_{1}^{\prime}\right| \varphi_{1, M}\right\rangle\left\langle\varphi_{1, M}\right|\right),
\end{aligned}
$$

where all $\lambda_{3}, \lambda_{2}, \lambda_{1}$, and $\lambda_{1}^{\prime}>0$.

It is interesting to compare the spin- 1 fermionic VBS state with the spin-1 bosonic VBS state of AKLT model. In the fermionic VBS state, the two-point spin-correlation function decays exponentially with a correlation length $\xi=1 / \ln 2$, longer than that for the AKLT model $(\xi=1 / \ln 3)$. Besides the obvious difference of the edge spin representation in an open chain, we can also see a sharp difference by computing the nonlocal string order parameter ${ }^{14,23}$ 


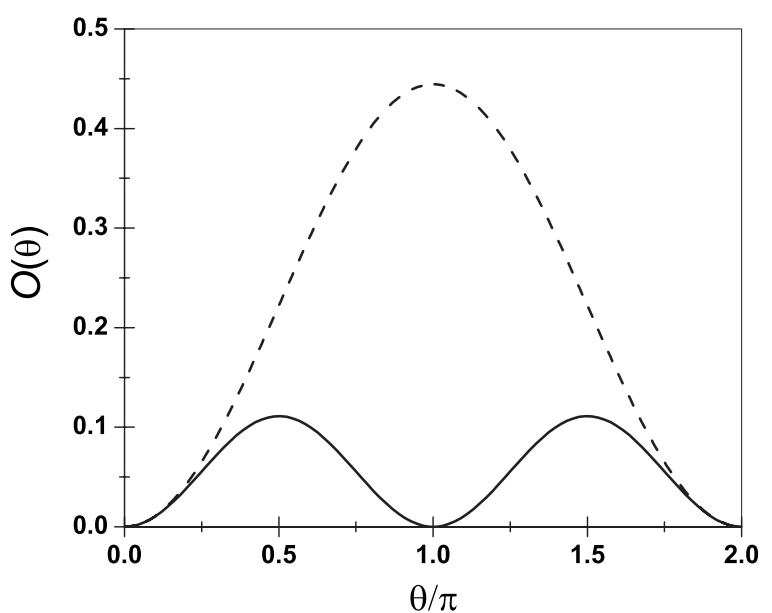

FIG. 3. The nonlocal string order parameter $\mathcal{O}(\theta)$ as a function of the spin-twist angle $\theta$ for the fermionic VBS state (solid line) and the bosonic VBS state (dashed line) of the AKLT model.

$$
\mathcal{O}(\theta)=\lim _{|j-i| \rightarrow \infty}\left\langle S_{i}^{z} \prod_{r=i}^{j-1} \exp \left(i \theta S_{r}^{z}\right) S_{j}^{z}\right\rangle .
$$

By using the transfer-matrix method, ${ }^{22}$ we arrive at the result $\mathcal{O}(\theta)=\frac{1}{9} \sin ^{2} \theta$ for the fermionic VBS state. For comparison, the values of the nonlocal order parameter $\mathcal{O}(\theta)$ for both the spin-1 fermionic VBS state and bosonic VBS state of the AKLT model are plotted in Fig. 3. For the spin-1 bosonic VBS state, $\mathcal{O}(\theta)$ reaches its maximum at $\theta=\pi$, which is reduced to the den Nijs-Rommelse string order parameter characterizing the hidden antiferromagnetic order in the AKLT VBS state. However, in the fermionic VBS state, $\mathcal{O}(\theta)$ has a minimum $\mathcal{O}(\pi)=0$ and the maximum value $1 / 9$ at both $\theta=\pi / 2$ and $3 \pi / 2$. This signifies that the hidden antiferromagnetic picture totally breaks down in the $S=1$ fermionic VBS state. How to describe such a state has not been clear so far.

\section{VBS states with an emergent $S O(2 S+1)$ symmetry}

In Sec. III A, we have mentioned an exceptional example: $S=2$ VBS states with virtual spin $J=3 / 2$. Besides the absent bond total spin $S_{T}=4$ channel of neighboring sites, there is a new forbidden channel $S_{T}=2$ in this VBS state. ${ }^{34}$ Therefore, its parent two-body Hamiltonian can be written as

$$
H=\sum_{i}\left[J_{1} P_{2}(i, i+1)+J_{2} P_{4}(i, i+1)\right],
$$

with $J_{1}, J_{2}>0$. According to Eq. (21), the projector Hamiltonian (30) can be rewritten as

$$
\begin{aligned}
H= & \sum_{\langle i j\rangle}\left[\frac{3 J_{2}-80 J_{1}}{84} \mathbf{S}_{i} \cdot \mathbf{S}_{j}+\frac{9 J_{2}-40 J_{1}}{360}\left(\mathbf{S}_{i} \cdot \mathbf{S}_{j}\right)^{2}\right. \\
& \left.+\frac{10 J_{1}+J_{2}}{60}\left(\mathbf{S}_{i} \cdot \mathbf{S}_{j}\right)^{3}+\frac{20 J_{1}+J_{2}}{2520}\left(\mathbf{S}_{i} \cdot \mathbf{S}_{j}\right)^{4}\right] .
\end{aligned}
$$

In fact, the $S=2$ VBS state with virtual spin $J=3 / 2$ has a hidden $S O(5)$ symmetry and its matrix product form was studied by Scalapino et $a l .{ }^{40}$ in an $S O(5)$ symmetric ladder system of interacting electrons.

The quantum spin-2 Hamiltonian in Eq. (30) belongs to a distinct class of exactly solvable quantum integer-spin chains presented by the first three of us very recently. ${ }^{34}$ The ground states of these Hamiltonians are $S O(2 S+1)$ symmetric matrix product states and exhibit hidden topological order. For $S$ $=1$, the $S O(3)$ symmetric matrix product state becomes the VBS state of spin-1 AKLT model. For $S=2$, the $S O(5)$ symmetric matrix product state is the $S=2 \mathrm{VBS}$ state with virtual spin $J=3 / 2$. However, it was not clear whether this family of matrix product states has a valence-bond picture for $S \geq 3$. By using the framework in Sec. II, we will show that there is indeed a VBS picture for these matrix product states. Actually, the virtual particles in these VBS states transform under the $2^{S}$-dimensional spinor representation of $S O(2 S+1)$.

It is convenient to promote the symmetry of the system and demand the spin- $S$ states on each site transform under the $(2 S+1)$-dimensional vector representation of $S O(2 S+1)$. The tensor product of two $S O(2 S+1)$ vectors on the adjacent sites can be decomposed as

$$
\underline{2 S+1} \otimes \underline{2 S+1}=\underline{1} \oplus \underline{S(2 S+1)} \oplus \underline{S(2 S+3)},
$$

where the number above each underline is the dimension of the corresponding IR. These $S O(2 S+1)$ IRs can be directly related to $S U(2)$ integer-spin IRs. Here 1 is the symmetric spin singlet, while the antisymmetric channel $S(2 S+1)$ and the symmetric channel $S(2 S+3)$ correspond to the total bond spin $S_{T}=1,3, \ldots, 2 S-\overline{1 \text { and } S_{T}}=2,4, \ldots, 2 S$ states, respectively. Therefore, the $S O(2 S+1)$ bond projection operators can be expressed using the spin projection operators as

$$
\begin{gathered}
P_{\underline{S(2 S+1)}}(i, j)=\sum_{l=1}^{S} P_{S_{T}=2 l-1}(i, j), \\
P_{\underline{S(2 S+3)}}(i, j)=\sum_{l=1}^{S} P_{S_{T}=2 l}(i, j) .
\end{gathered}
$$

On each lattice site, the $S O(2 S+1)$ vectors can be formed by tensor decomposition of two virtual $2^{S}$-dimensional spinors

$$
\underline{2^{S}} \otimes \underline{2^{S}}=\underset{q=0}{\oplus}\left(\begin{array}{c}
q \\
2 S+1
\end{array}\right),
$$

where $\left(\begin{array}{c}q \\ 2 S+1\end{array}\right)=\frac{(2 S+1) !}{q !(2 S-q+1) !}$. Note that $q=0$ and $q=1$ in Eq. (35) correspond to singlet representation and $(2 S+1)$-dimensional vector representation, respectively. For $S=1$, Eq. (35) recovers the well-known decomposition $\underline{2} \otimes \underline{2}=\underline{1} \oplus \underline{3}$ of two spin$1 / 2$ spinors. For $S=2$, Eq. (35) can be interpreted as the decomposition $\underline{4} \otimes \underline{4}=\underline{1} \oplus \underline{5} \oplus \underline{10}$, where the $S O(5)$ spinors can be viewed as spin-3/2 variables because $S O(5) \simeq S p(4)$. However, the $S O(2 S+1)$ spinors in Eq. (35) for $S \geq 3$ do not have $S U(2)$ spin counterparts.

Following the discussions in Sec. II, the $S O(2 S+1)$ symmetric VBS states can be constructed by combining the virtual spinors on the neighboring sites into valence-bond singlets. By comparing Eqs. (32) and (35), one finds that the IR channel $S(2 S+3)$ for any two neighboring sites is absent in 
these VBS states. Here an interesting observation is that those IR channels with $q \geq 2$ in Eq. (35) are actually absent for two adjacent sites due to the projection of two virtual spinors onto the physical vector representation in each site. Therefore, the $S O(2 S+1)$-invariant parent Hamiltonian for the $S O(2 S+1)$ symmetric VBS states is given by

$$
H=\sum_{i} P_{\underline{S(2 S+3)}}(i, i+1) .
$$

Since the null space of these VBS states is the nonzero even total spin channels, we can extend the $S O(2 S+1)$-invariant parent Hamiltonian to the following $S U(2)$-invariant quantum integer-spin Hamiltonian:

$$
H=\sum_{i} \sum_{l=1}^{S} J_{l} P_{S_{T}=2 l}(i, i+1),
$$

with all $J_{l}>0$.

Actually, the $S O(2 S+1)$ symmetric VBS states are equivalent to the matrix product states studied in Ref. 34. In the present VBS form, the origin of emergent $S O(2 S+1)$ symmetry and the $2^{S}$ edge states on each boundary of an open chain are quite clear. Although the edge degrees of freedom in $S=1$ and $S=2$ cases can be viewed as $S U(2)$ spin variables, they transform under $S O(2 S+1)$ spinor representation for $S \geq 3$ cases. It is interesting to compare these $S O(2 S+1)$ symmetric VBS states to the spin- $S$ VBS states formed by virtual spin $J=\left(2^{S}-1\right) / 2$ in Sec. III A. Although they are both unique in a periodic chain and $4^{S}$-fold degenerate in an open chain, their distinct edge states show that they belong to two different topological classes. These explicit examples imply that the ground-state degeneracy is not sufficient to characterize the topologically ordered states.

\section{IV. $S O(5)$ SYMMETRIC VBS STATES}

So far, we are restricted to the case of $S U(2)$ integer spin in each site. Actually, the method discussed in Sec. II can be applied for a general Lie group $G$; we thus move on to $S O(5)$ Lie group, where the physical states transform under $S O(5)$ IRs.

The $S O(5)$ Lie algebra has ten generators $L^{a b}(1 \leq a<b$ $\leq 5)$ satisfying the commutation relations

$$
\left[L^{a b}, L^{c d}\right]=i\left(\delta_{a d} L^{b c}+\delta_{b c} L^{a d}-\delta_{a c} L^{b d}-\delta_{b d} L^{a c}\right) .
$$

Mathematically, the IRs of $S O(5)$ are labeled by two integers $(p, q)$, with $p \geq q \geq 0$. For the $(p, q)$ representation of $S O(5)$ Lie group, the dimensionality $d(p, q)$ and the Casimir charge $C(p, q)$ are given by ${ }^{41}$

$$
\begin{gathered}
d(p, q)=(1+q)(1+p-q)\left(1+\frac{p}{2}\right)\left(1+\frac{p+q}{3}\right), \\
C(p, q)=\sum_{a<b}\left(L^{a b}\right)^{2}=\frac{p^{2}}{2}+\frac{q^{2}}{2}+2 p+q,
\end{gathered}
$$

respectively. The dimensionality and Casimir charge for the simplest $S O(5)$ irreducible representations are listed in Table I.
TABLE I. Several irreducible representations of the $S O(5) \mathrm{Lie}$ group.

\begin{tabular}{ccc}
\hline \hline Representation & Dimension & Casimir charge \\
\hline$(0,0)$ & 1 & 0 \\
$(1,0)$ & 4 & $5 / 2$ \\
$(1,1)$ & 5 & 4 \\
$(2,0)$ & 10 & 6 \\
$(2,2)$ & 14 & 10 \\
$(3,1)$ & 35 & 12 \\
$(4,0)$ & 35 & 16 \\
\hline \hline
\end{tabular}

\section{A. Bosonic $S O(5)$ VBS states}

We begin with the ten-dimensional $(2,0)$ adjoint representation of $S O(5)$. The bosonic $S O(5) / S p(4)$ VBS state of this system was first considered by Schuricht and Rachel. ${ }^{37}$ Their strategy is to construct the $(2,0)$ adjoint representation by two virtual particles transforming under the $(1,0)$ spinor representation,

$$
(1,0) \otimes(1,0)=(0,0) \oplus(1,1) \oplus(2,0),
$$

where $(0,0)$ and $(1,1)$ are antisymmetric and $(2,0)$ is the only symmetric product representation. Therefore, one can obtain the physical $(2,0)$ adjoint representation by endowing bosonic statistics to the virtual $(1,0)$ spinor particles. This is analogous to the $S U(2)$ Schwinger boson representation that symmetrizes two spin-1/2 spinors to construct a spin-1 representation. Using the four-component $S O(5)$ Schwinger bosons, the $S O(5)$ generators in Eq. (38) can be defined as

$$
L^{a b}=-\frac{1}{2} \sum_{\mu, \nu=1}^{4} b_{\mu}^{\dagger} \Gamma_{\mu \nu}^{a b} b_{\nu},
$$

where $\Gamma^{a b}=\left[\Gamma^{a}, \Gamma^{b}\right] / 2 i$ and

$$
\Gamma^{1,2,3}=\left(\begin{array}{cc}
0 & i \vec{\sigma} \\
-i \vec{\sigma} & 0
\end{array}\right), \quad \Gamma^{4}=\left(\begin{array}{cc}
0 & I \\
I & 0
\end{array}\right), \quad \Gamma^{5}=\left(\begin{array}{cc}
I & 0 \\
0 & -I
\end{array}\right) .
$$

For the $(2,0)$ adjoint representation with $\sum_{\mu=1}^{4} b_{\mu}^{\dagger} b_{\mu}=2$, the ten states in a bosonic language are shown in the $(2,0)$ weight diagram in Fig. 4. After a rotation by $45^{\circ}$, this weight diagram is identical to that given by Schuricht and Rachel. Here we choose the Clifford algebra generated by the $\Gamma$ matrices to define the $S O(5)$ generators. The advantage of our convention is to find an interesting nonlocal hidden string order in the $(2,0)$ bosonic VBS state below.

The $(2,0)$ bosonic $S O(5)$ VBS state is formed by contracting two $(1,0)$ spinors on neighboring sites into a valencebond $S O(5)$ singlet. Its wave function can be written compactly as

$$
\left|\Psi_{2}\right\rangle=\prod_{i}\left(\sum_{\mu \nu} b_{i, \mu}^{\dagger} R_{\mu \nu} b_{i+1, \nu}^{\dagger}\right)|v\rangle,
$$

where the antisymmetric matrix $R$ is given by 


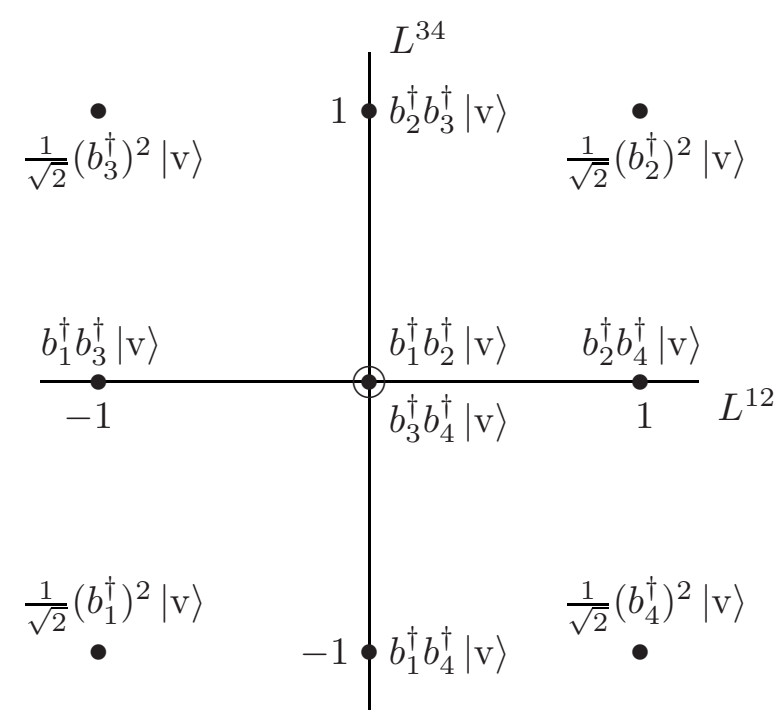

FIG. 4. Weight diagram and the bosonic realization of the $(2,0)$ adjoint representation of $S O(5)$. There is a twofold degeneracy with $L^{12}=L^{34}=0$.

$$
R=\left(\begin{array}{cc}
-i \sigma^{y} & 0 \\
0 & -i \sigma^{y}
\end{array}\right)
$$

with the following properties:

$$
\begin{gathered}
R^{2}=-1, \quad R^{\dagger}=R^{-1}=R^{T}=-R, \\
R \Gamma^{a} R^{-1}=\left(\Gamma^{a}\right)^{T}, \quad R \Gamma^{a b} R^{-1}=-\left(\Gamma^{a b}\right)^{T} .
\end{gathered}
$$

The tensor product decomposition of two neighboring $(2,0)$ adjoint representations is written as

$$
\begin{aligned}
(2,0) \otimes(2,0)= & (0,0) \oplus(1,1) \oplus(2,0) \oplus(2,2) \oplus(3,1) \\
& \oplus(4,0) .
\end{aligned}
$$

In the $(2,0)$ bosonic VBS state, a valence-bond singlet of two virtual $(1,0)$ spinors are created and therefore the two adjacent sites can only transform $(0,0),(1,1)$, and $(2,0)$ representations according to Eq. (41). Consequently, $\left|\Psi_{2}\right\rangle$ is an exact ground state of the projector Hamiltonian

$$
H=\sum_{i}\left[J_{1} P_{(2,2)}(i, i+1)+J_{2} P_{(3,1)}(i, i+1)+J_{3} P_{(4,0)}(i, i+1)\right],
$$

where $J_{1}, J_{2}$, and $J_{3}>0$ and $P_{(2,2)}, P_{(3,1)}$, and $P_{(4,0)}$ are projectors onto the $(2,2),(3,1)$, and $(4,0)$ representations, respectively.

Furthermore, the $(2,0)$ bosonic VBS state contain a welldefined hidden string order. This can be observed in its matrix product wave function with the local matrix

$$
g_{i}=\left(\begin{array}{cccc}
|0,0\rangle & \sqrt{2}|1,1\rangle & |0,1\rangle & |1,0\rangle \\
-\sqrt{2}|-1,-1\rangle & -|0,0\rangle & -|-1,0\rangle & -|0,-1\rangle \\
|0,-1\rangle & |1,0\rangle & |0,0\rangle^{\prime} & \sqrt{2}|1,-1\rangle \\
-|-1,0\rangle & -|0,1\rangle & -\sqrt{2}|-1,1\rangle & -|0,0\rangle^{\prime}
\end{array}\right)_{i}
$$

where we take $|0,0\rangle=b_{1}^{\dagger} b_{2}^{\dagger}|v\rangle$ and $|0,0\rangle^{\prime}=b_{3}^{\dagger} b_{4}^{\dagger}|v\rangle$. In both of the $m_{1}$ and $m_{2}$ channels, it can be shown that $\left|m_{\eta}\right\rangle(\eta$ $=1,2$ ) has a hidden antiferromagnetic order. In other word, the states of $m_{\eta}=1$ and -1 will alternate in space if all the $m_{\eta}=0$ states between them are ignored. A typical configuration of this state is given by

$$
\begin{aligned}
& m_{1}: \cdots 0 \uparrow 00 \downarrow \uparrow \downarrow 000 \uparrow 0 \downarrow 0 \uparrow \cdots \\
& m_{2}: \cdots 0 \uparrow 000 \downarrow \uparrow 0 \downarrow 00 \uparrow \downarrow 00 \cdots
\end{aligned}
$$

where $(\uparrow, 0, \downarrow)$ represent $|m\rangle=(|1\rangle,|0\rangle,|-1\rangle)$. This hidden antiferromagnetic order is in analogy with the spin-1 VBS state of AKLT model ${ }^{12}$ and its $S O(2 S+1)$ generalization. ${ }^{34}$ To characterize this hidden antiferromagnetic order, one can generalize the den Nijs-Rommelse string order parameters as

$$
\mathcal{O}^{a b}=\lim _{|j-i| \rightarrow \infty}\left\langle L_{i}^{a b} \prod_{r=i}^{j-1} \exp \left(i \pi L_{r}^{a b}\right) L_{j}^{a b}\right\rangle .
$$

In fact, the nonlocal string order parameters for the Cartan generators introduced by Schuricht and Rachel ${ }^{37}$ are combinations of our $\mathcal{O}^{12}$ and $\mathcal{O}^{34}$. The advantage of our convention is that the string order parameters in Eq. (49) clearly reflect a hidden antiferromagnetic order in the $(2,0)$ bosonic $S O(5)$ VBS state. The value of these string order parameters can be obtained by a probability argument. These nonlocal string order parameters should all be equal to each other because the VBS state preserves $S O(5)$ symmetry. Thus, we only need to evaluate the value of $\mathcal{O}^{12}$ by considering the $m_{1}$ channel. The role of the nonlocal string phase factor in Eq. (49) is to correlate the finite spin-polarized states in the $m_{1}$ channel at the two ends of the string. If nonzero $m_{1}$ takes the same value at the two ends, then the phase factor is equal to 1. On the other hand, if a nonzero $m_{1}$ takes two different values at the two ends, then the phase factor is equal to -1 . Thus, the value of $\mathcal{O}^{12}=9 / 25$ is a square of the probability of the nonzero $m_{1}= \pm 1$ appearing at the ends of the string. Correspondingly, a generalized Kennedy-Tasaki unitary transformation can be designed according to Ref. 34 and we expect that the $S O(5)$ symmetry of the original Hamiltonian is reduced to $\left(Z_{2} \times Z_{2}\right)^{2}$ under such a nonlocal transformation. The nonlocal string order parameters in Eq. (49) for the Cartan generators will be transformed to two-point correlation functions, which properly characterize the hidden $\left(Z_{2}\right.$ $\left.\times Z_{2}\right)^{2}$ symmetry breaking. Thus, in this state, the nonlocal string order and the 16-fold degeneracy in an open chain can be viewed as natural consequences of a hidden $\left(Z_{2} \times Z_{2}\right)^{2}$ symmetry breaking.

In fact, the bosonic $S O(5)$ VBS state of $(2,0)$ adjoint representation can be generalized to the totally symmetric $(p, 0)$ representation with even $p$. Namely, $(p, 0)$ representation for even $p$ can be constructed by two $(p / 2,0)$ representations. However, there is an alternative way to take the advantage of 
a generalized Schwinger boson representation. Using the generalized Schwinger boson representation, the $(p, 0)$ representation in each site can be constructed by symmetrization of $p$ spinors and the local constraint is now replaced with $\sum_{\mu=1}^{4} b_{\mu}^{\dagger} b_{\mu}=p$. Thus, the bosonic $S O(5) \mathrm{VBS}$ states for $(p, 0)$ representation can be written as

$$
\left|\Psi_{3}\right\rangle=\prod_{i}\left(\sum_{\mu \nu} b_{i, \mu}^{\dagger} R_{\mu \nu} b_{i+1, \nu}^{\dagger}\right)^{p / 2}|v\rangle .
$$

In an open chain, there are fractionalized edge states transforming under $(p / 2,0)$ representation. Once a $\mathrm{CP}^{3}$ coherent state representation ${ }^{36}$ is used, the $(p, 0)$ VBS states have the Jastrow form. They are analogous to the fractional quantum Hall states in $\mathrm{CP}^{3}$ space ${ }^{42}$ at filling fraction $\nu=2 / p$, in the same sense as the resemblance ${ }^{11}$ between VBS states of AKLT model and the fractional quantum Hall states in spherical geometry.

Since the tensor product decomposition of two $(p, 0)$ representations is given by

$$
(p, 0) \otimes(p, 0)=\sum_{k=0}^{p} \sum_{l=0}^{k}(k+l, k-l)
$$

and $p / 2$ valence-bond singlets are created between adjacent sites in $\left|\Psi_{3}\right\rangle$, the only finite projection on two adjacent sites is given by

$$
(p / 2,0) \otimes(p / 2,0)=\sum_{k=0}^{p / 2} \sum_{l=0}^{k}(k+l, k-l) .
$$

Thus, the null space of the two-site-reduced density matrix is given by a sum of the representations written as $\sum_{k=p / 2+1}^{p} \sum_{l=0}^{k}(k+l, k-l)$ and the corresponding parent Hamiltonian of $\left|\Psi_{3}\right\rangle$ is given by

$$
H=\sum_{i} \sum_{k=p / 2+1}^{p} \sum_{l=0}^{k} J_{(k+l, k-l)} P_{(k+l, k-l)}(i, i+1)
$$

where all $J_{(k+l, k-l)}>0$ and $P_{(k+l, k-l)}$ is the projection operator onto the $(k+l, k-l)$ representation states.

With the help of the Casimir charge in Eq. (40), the $S O(5)$ projectors can be written as polynomial functions of $S O(5)$ generators. According to Eq. (51), these projectors satisfy a completeness relation

$$
\sum_{k=0}^{p} \sum_{l=0}^{k} P_{(k+l, k-l)}(i, j)=1 .
$$

Considering the two-site Casimir charge $\Sigma_{a<b}\left(L_{i}^{a b}+L_{j}^{a b}\right)^{2}$, we can write the $S O(5)$ Heisenberg interaction as

$$
\sum_{a<b} L_{i}^{a b} L_{j}^{a b}=\frac{1}{2} \sum_{k=0}^{p} \sum_{l=0}^{k}\left[C(k+l, k-l)-\left(p^{2}+4 p\right)\right] P_{(k+l, k-l)}(i, j) .
$$

Using the properties of the projectors, we have

$$
\begin{aligned}
& \frac{1}{2}\left(c_{1}^{\dagger}+i c_{2}^{\dagger}\right)\left(c_{3}^{\dagger}-i c_{4}^{\dagger}\right)|\mathrm{v}\rangle \stackrel{\substack{L^{34} \\
\frac{1}{\sqrt{2}}\left(c_{3}^{\dagger}-i c_{4}^{\dagger}\right) c_{5}^{\dagger}|\mathrm{v}\rangle \\
\bullet}}{\frac{1}{2}\left(c_{1}^{\dagger}-i c_{2}^{\dagger}\right)\left(c_{3}^{\dagger}-i c_{4}^{\dagger}\right)|\mathrm{v}\rangle} \\
& \underset{-1}{\frac{1}{\sqrt{2}}\left(c_{1}^{\dagger}+i c_{2}^{\dagger}\right) c_{5}^{\dagger}|\mathrm{v}\rangle} \quad \underbrace{\frac{1}{\sqrt{2}}\left(c_{1}^{\dagger}-i c_{2}^{\dagger}\right) c_{5}^{\dagger}|\mathrm{v}\rangle}_{c_{3}^{\dagger} c_{2}^{\dagger}|\mathrm{v}\rangle} L^{12} \\
& \frac{1}{2}\left(c_{1}^{\dagger}+i c_{2}^{\dagger}\right)\left(c_{3}^{\dagger}+i c_{4}^{\dagger}\right)|\mathrm{v}\rangle{ }_{-1}^{\bullet} \biguplus_{\frac{1}{\sqrt{2}}\left(c_{3}^{\dagger}+i c_{4}^{\dagger}\right) c_{5}^{\dagger}|\mathrm{v}\rangle} \frac{1}{2}\left(c_{1}^{\dagger}-i c_{2}^{\dagger}\right)\left(c_{3}^{\dagger}+i c_{4}^{\dagger}\right)|\mathrm{v}\rangle
\end{aligned}
$$

FIG. 5. Weight diagram and the fermionic realization of the $(2,0)$ adjoint representation of $S O(5)$ Lie algebra.

$$
\begin{aligned}
& \left(\sum_{a<b} L_{i}^{a b} L_{j}^{a b}\right)^{n} \\
& \quad=\frac{1}{2^{n}} \sum_{k=0}^{p} \sum_{l=0}^{k}\left[C(k+l, k-l)-\left(p^{2}+4 p\right)\right]^{n} P_{(k+l, k-l)}(i, j) .
\end{aligned}
$$

Together with the completeness relation (54), this formula can be inverted, so that each projector can be represented by a polynomial function of $S O(5)$ Heisenberg interaction $\sum_{a<b} L_{i}^{a b} L_{j}^{a b}$.

\section{B. Fermionic $S O(5)$ VBS state}

In this subsection, we present another way to construct the $(2,0)$ adjoint representation, i.e., by using two $(1,1)$ vector representations,

$$
(1,1) \otimes(1,1)=(0,0) \oplus(2,0) \oplus(2,2),
$$

where the $(2,0)$ adjoint representation is antisymmetric and $(0,0)$ and $(2,2)$ are symmetric. This is because the orthogonal groups have a general property that the adjoint representation is the only resulting antisymmetric channel of two vector representations. ${ }^{34}$ The simplest realization of this property is the $S O(3)$ spin-1 case discussed in Sec. III B, where the antisymmetrization of two vector spin-1 representations only yields the spin-1 adjoint representation.

If we use the fermionic statistics to implement the antisymmetrization, the ten states in the adjoint representation can be written as $c_{a}^{\dagger} c_{b}^{\dagger}|v\rangle$, where $1 \leq a<b \leq 5$. Moreover, the $S O(5)$ generators are defined by

$$
L^{a b}=i\left(c_{a}^{\dagger} c_{b}-c_{b}^{\dagger} c_{a}\right)
$$

and a double occupancy constraint $\sum_{a=1}^{5} c_{a}^{\dagger} c_{a}=2$ can guarantee the adjoint representation in each lattice site. Using these fermionic variables, the $(2,0)$ weight diagram is shown in Fig. 5. 
Using the fermion variables, the $(2,0)$ fermionic VBS state with two virtual $(1,1)$ vector $S O(5)$ representations can be written as

$$
\left|\Psi_{4}\right\rangle=\prod_{i}\left(\sum_{a} c_{i, a}^{\dagger} c_{i+1, a}^{\dagger}\right)|v\rangle .
$$

In an open chain, the edge spins transform under $(1,1)$ vector $S O(5)$ representation, different from the $(1,0)$ spinor $S O(5)$ representation in the $(2,0)$ bosonic $S O(5)$ VBS state. Another interesting observation is that the perfect nonlocal string order presence in the $(2,0)$ bosonic $S O(5)$ VBS state vanishes in the fermionic VBS state, because the string order parameter (49) for this state is found to be zero. In this sense, the bosonic and fermionic $(2,0)$ VBS states can be viewed as $S O(5)$ generalizations of spin-1 VBS states of AKLT model and fermionic VBS state in Sec. III B.

Finally, using two-body interactions, one can construct the parent Hamiltonian for this fermionic $S O(5)$ VBS state. Since any two adjacent sites can only transform under $(0,0)$, $(2,0)$, and $(2,2)$ representations, $\left|\Psi_{4}\right\rangle$ is an exact zero-energy ground state of the projector Hamiltonian

$$
\begin{aligned}
H= & \sum_{i}\left[K_{1} P_{(1,1)}(i, i+1)+K_{2} P_{(3,1)}(i, i+1)\right. \\
& \left.+K_{3} P_{(4,0)}(i, i+1)\right],
\end{aligned}
$$

for $K_{1}, K_{2}$, and $K_{3}>0$. The possible hidden order is still under investigation.

\section{CONCLUSION}

In conclusion, we have presented a general method to construct one-dimensional VBS states embedded with Lie group $G$ and their parent Hamiltonians. This provides examples that the topologically ordered states can be systematically generated in one dimension and are characterized by their edge state representations as well as their ground-state degeneracy.
For quantum integer-spin- $S$ chains, there exists two topologically distinct families: (i) the virtual particles transform under $S U(2)$ spin- $J$ representations and (ii) the virtual particles are $S O(2 S+1)$ spinors. In the first class, a spin-1 fermionic VBS state is constructed as an explicit example. Compared to the celebrated $S=1$ valence-bond solid state of AKLT model, the fermionic valence-bond solid state shows drastic differences on the edge states and hidden string order. For the second class, it has been shown that these valencebond solid states with an emergent $S O(2 S+1)$ symmetry are equivalent to the previously proposed $S O(2 S+1)$ symmetric matrix product states. ${ }^{34}$ The present formalism explicitly displays that the edge states of an open chain transform under the $S O(2 S+1) 2^{S}$-dimensional spinor representation.

To generalize the VBS states in $S U(2)$ symmetric quantum integer-spin chains, two types of VBS states with the $S O(5)$ symmetry are considered including (i) bosonic $S O(5)$ VBS states formed by a symmetrization of two spinor representations in each site and (ii) a fermionic $S O(5)$ VBS state with $(2,0)$ adjoint representation formed by antisymmetrization of two vector representations.

It can be expected that the ideas and formalism developed in this work are very useful and can be generalized to the tensor product states (projected entangled pair states) for higher dimensional correlated systems. ${ }^{43}$ The understanding of the physical properties of these states is the first step to characterize higher dimensional topological states, which certainly deserves further investigations.

\section{ACKNOWLEDGMENTS}

One of the authors (H.H.T.) would like to thank Mikel Sanz and J. Ignacio Cirac for stimulating discussions during the visit to the Max Planck Institute for Quantum Optics. He is also grateful to Stephan Rachel for several helpful suggestions. We acknowledge the support of NSF-China and the National Program for Basic Research of MOST-China. *gmzhang@mail.tsinghua.edu.cn

${ }^{1}$ X.-G. Wen, Quantum Field Theory of Many-Body Systems (Oxford University Press, Oxford, 2004).

${ }^{2}$ A. Kitaev, Ann. Phys. (N.Y.) 321, 2 (2006).

${ }^{3}$ X.-Y. Feng, G.-M. Zhang, and T. Xiang, Phys. Rev. Lett. 98, 087204 (2007).

${ }^{4}$ M. Oshikawa and T. Senthil, Phys. Rev. Lett. 96, 060601 (2006).

${ }^{5}$ H. Bombin and M. A. Martin-Delgado, Phys. Rev. B 75, 075103 (2007).

${ }^{6}$ M. Aguado and G. Vidal, Phys. Rev. Lett. 100, 070404 (2008).

${ }^{7}$ D. Pérez-García, M. M. Wolf, M. Sanz, F. Verstraete, and J. I. Cirac, Phys. Rev. Lett. 100, 167202 (2008).

${ }^{8}$ X. G. Wen, Int. J. Mod. Phys. B 4, 239 (1990); X. G. Wen and Q. Niu, Phys. Rev. B 41, 9377 (1990); Adv. Phys. 44, 405 (1995).

${ }^{9}$ F. D. M. Haldane, Phys. Lett. 93A, 464 (1983); Phys. Rev. Lett. 50, 1153 (1983).
${ }^{10}$ I. Affleck, T. Kennedy, E. H. Lieb, and H. Tasaki, Phys. Rev. Lett. 59, 799 (1987); Commun. Math. Phys. 115, 477 (1988).

${ }^{11}$ D. P. Arovas, A. Auerbach, and F. D. M. Haldane, Phys. Rev. Lett. 60, 531 (1988).

${ }^{12}$ M. den Nijs and K. Rommelse, Phys. Rev. B 40, 4709 (1989).

${ }^{13}$ T. Kennedy and H. Tasaki, Phys. Rev. B 45, 304 (1992); Commun. Math. Phys. 147, 431 (1992).

${ }^{14}$ M. Oshikawa, J. Phys.: Condens. Matter 4, 7469 (1992).

${ }^{15}$ T.-K. Ng, Phys. Rev. B 50, 555 (1994).

${ }^{16}$ S.-J. Qin, T.-K. Ng, and Z.-B. Su, Phys. Rev. B 52, 12844 (1995).

${ }^{17}$ M. Hagiwara, K. Katsumata, I. Affleck, B. I. Halperin, and J. P. Renard, Phys. Rev. Lett. 65, 3181 (1990).

${ }^{18}$ S. H. Glarum, S. Geschwind, K. M. Lee, M. L. Kaplan, and J. Michel, Phys. Rev. Lett. 67, 1614 (1991).

${ }^{19}$ F. Tedoldi, R. Santachiara, and M. Horvatić, Phys. Rev. Lett. 83, 412 (1999). 
${ }^{20}$ G. Xu, G. Aeppli, M. E. Bisher, C. Broholm, J. F. DiTusa, C. D. Frost, T. Ito, K. Oka, R. L. Paul, H. Takagi, and M. M. J. Treacy, Science 289, 419 (2000).

${ }^{21}$ M. Fannes, B. Nachtergaele, and R. F. Werner, Europhys. Lett. 10, 633 (1989); Commun. Math. Phys. 144, 443 (1992).

${ }^{22}$ A. Klümper, A. Schadschneider, and J. Zittartz, J. Phys. A 24, L955 (1991); Z. Phys. B Condens. Matter 87, 281 (1992).

${ }^{23}$ K. Totsuka and M. Suzuki, J. Phys.: Condens. Matter 7, 1639 (1995).

${ }^{24}$ S. Östlund and S. Rommer, Phys. Rev. Lett. 75, 3537 (1995); S. Rommer and S. Östlund, Phys. Rev. B 55, 2164 (1997).

${ }^{25}$ S. R. White, Phys. Rev. Lett. 69, 2863 (1992).

${ }^{26}$ G. Vidal, Phys. Rev. Lett. 93, 040502 (2004).

${ }^{27}$ J. Eisert, M. Cramer, and M. B. Plenio, arXiv:0808.3773, Rev. Mod. Phys. (to be published).

${ }^{28}$ F. Verstraete, M. A. Martín-Delgado, and J. I. Cirac, Phys. Rev. Lett. 92, 087201 (2004).

${ }^{29}$ H. Fan, V. E. Korepin, and V. Roychowdhury, Phys. Rev. Lett. 93, 227203 (2004).

${ }^{30}$ H. Katsura, T. Hirano, and Y. Hatsugai, Phys. Rev. B 76, 012401 (2007).

${ }^{31}$ F. Verstraete and J. I. Cirac, Phys. Rev. A 70, 060302(R) (2004).
${ }^{32}$ G. K. Brennen and A. Miyake, Phys. Rev. Lett. 101, 010502 (2008).

${ }^{33}$ D. Pérez-García, F. Verstraete, M. M. Wolf, and J. I. Cirac, Quantum Inf. Comput. 7, 401 (2007).

${ }^{34}$ H.-H. Tu, G.-M. Zhang, and T. Xiang, J. Phys. A 41, 415201 (2008); Phys. Rev. B 78, 094404 (2008).

${ }^{35}$ M. Greiter, S. Rachel, and D. Schuricht, Phys. Rev. B 75, 060401(R) (2007); M. Greiter and S. Rachel, ibid. 75, 184441 (2007).

${ }^{36}$ D. P. Arovas, Phys. Rev. B 77, 104404 (2008).

${ }^{37}$ D. Schuricht and S. Rachel, Phys. Rev. B 78, 014430 (2008).

${ }^{38}$ A. K. Kolezhuk and H. J. Mikeska, Int. J. Mod. Phys. B 12, 2325 (1998); M. Asoudeh, V. Karimipour, and A. Sadrolashrafi, Phys. Rev. B 75, 224427 (2007).

${ }^{39}$ M. Sanz, M. M. Wolf, D. Perez-Garcia, and J. I. Cirac, Phys. Rev. A 79, 042308 (2009).

${ }^{40}$ D. Scalapino, S.-C. Zhang, and W. Hanke, Phys. Rev. B 58, 443 (1998).

${ }^{41}$ S.-C. Zhang and J.-P. Hu, Science 294, 823 (2001).

${ }^{42}$ S.-C. Zhang, Phys. Rev. Lett. 90, 196801 (2003).

${ }^{43}$ H.-C. Jiang, Z.-Y. Weng, and T. Xiang, Phys. Rev. Lett. 101, 090603 (2008). 